\title{
Article \\ Transformations of Multi-Player Normal form Games by Preplay Offers between Players
}

\author{
Valentin Goranko $1,2,+(\mathbb{D}$
}

Citation: Goranko, V.

Transformations of Multi-Player

Normal form Games by Preplay

Offers between Players. Axioms 2022,

11, 73. https://doi.org/10.3390/

axioms11020073

Academic Editor: Valery Y. Glizer

Received: 10 January 2022

Accepted: 4 February 2022

Published: 12 February 2022

Publisher's Note: MDPI stays neutral with regard to jurisdictional claims in published maps and institutional affiliations.

Copyright: (c) 2022 by the author. Licensee MDPI, Basel, Switzerland. This article is an open access article distributed under the terms and conditions of the Creative Commons Attribution (CC BY) license (https:// creativecommons.org/licenses/by/ $4.0 /)$.
1 Department of Philosophy, Stockholm University, 10691 Stockholm, Sweden; valentin.goranko@philosophy.su.se

2 School of Mathematics, University of the Witwatersrand, Johannesburg 2000, South Africa

+ Visiting professorship at the University of the Witwatersrand.

\begin{abstract}
The paper deals with multiplayer normal form games which are preceded by a 'preplay negotiation phase' consisting of exchange of preplay offers by players for payments of utility to other players conditional on them playing designated in the offers strategies. The game-theoretic effect of such preplay offers is a transformation of the payoff matrix of the game, obtained by transferring the offered payments between the payoffs of the respective players; thus, certain groups of game matrix transformations naturally emerge. The main result is an explicit and rather transparent algebraic characterization of the possible transformations of the payoff matrix of any given $N$ person normal form game induced by preplay offers for transfer of payments. That result can be used to describe the 'bargaining space' of the game and to determine the mutually optimal game transformations that rational players can achieve by exchange of preplay offers.
\end{abstract}

Keywords: normal form games; preplay offers; game transformations; groups of game matrix transformations

MSC: 91A05; 91A06; 91A10; 15A30; 15B99

\section{Introduction}

Some normal form games have no pure strategy Nash equilibria, while others, such as the Prisoners' Dilemma, have rather unsatisfactory ones, i.e., they are strongly Paretodominated. Sometimes, more mutually beneficial outcomes can be achieved if players could communicate before the play of the game, to make binding offers for payments of bonuses to other players to provide additional incentives for them to play as desired by offering players strategies. More precisely, I assume the possibility that before playing the game, any player A can make a binding offer to any other player B to pay them. After the game is played, a declared amount of utility $\delta$ is awarded if B plays strategy s, as specified in the offer by $A$.

Every player can make several such preplay offers (possibly to different players), and I only consider offers contingent on pure strategies, even though players can still play mixed strategies. Any preplay offer of player A is binding and irrevocable for $A$, and it is contingent on B playing the strategy specified by A. However, such an offer does not create any obligation for $B$ (and, therefore, does not transform the game into a cooperative one), as $B$ remains free to choose their strategy when the game is actually played. The key point here is that every preplay offer transforms the given normal form game into another one by an explicitly defined transformation of the payoff matrix.

By exchanging preplay offers of payments, the players create a preliminary negotiation phase essentially structured as an extensive-form bargaining game. Such scenarios arise in a wide spectrum of economic, social, and political situations, such as collusions, compensations, incentives, concessions, compromises, and other kinds of deals in economic and political negotiations, out-of-court settlements of legal cases, and corruption schemes. 
Normal form games with preplay negotiation phase consisting of exchange of preplay offers were introduced and studied in [1-3], where some results analyzing efficiency of preplay negotiations on such game transformations between rational players were obtained. In the present paper, I study the purely mathematical effect of preplay offers on the payoff matrices of normal form games, disregarding any rationality considerations that may prescribe to players if and what offers are available to make. That is, here we are only interested in how a game matrix can be transformed by preplay offers, but not why players may wish to exchange offers inducing a given transformation. Our objective is to obtain an explicit algebraic characterization of all possible offer-induced transformations of a given normal form game. Thus, the present work is a technical counterpart to the truly game theoretic study initiated in the above-referred papers. In the concluding section, I briefly discuss the game-theoretic use and impact of our results.

\subsection{Contributions}

The contributions of this paper are as follows:

1. Introduction and initial study of offer-induced game matrix transformation. I show, inter alia, that the set of such transformations on the class of all game payoff matrices of given dimension forms a group with respect to composition of transformations;

2. Complete and transparent characterization of the orbit of any game payoff matrix under that group of transformations, i.e., explicit characterization of the class of all normal form games that can be obtained from a given game matrix by means of offer-induced transformations. I first obtain such characterization for two-person games (Theorem 1), and then extend the result to $N$-person games (Theorem 3 ). While the proofs are standard applications of linear algebra, I argue that the characterizations themselves are quite useful from game-theoretic perspective as they indicate to the players how they can possibly transform the game they are playing by exchanging suitable preplay offers; thus, describing their "bargaining space" for that game;

3. Proof that any such transformation of a game payoff matrix $M$ can be obtained by choosing any strategy profile in $M$ and setting suitable payoffs satisfying a certain condition for all outcomes obtained by allowing any one, and only one, player to deviate from that strategy profile, by using recurrent formulae for computing the remaining payoffs in the transformed matrix.

\subsection{Related Work}

The formal theory of bargaining goes back to Nash [4] and Harsanyi [5]. Since then, there were extensive studies of negotiations and bargaining in cooperative and noncooperative games, e.g., surveys in [6]. The preplay negotiation games studied here are closely related in spirit to bargaining games, see [7-11] and were introduced and studied in [1-3], which are precursors and most closely related publications to the present work. The paper [1] introduces the extension of normal form games with a preplay negotiations phase, where players can exchange preplay offers that are conditional on suggested matching offers of the same kind made in return by the receiver. The follow-up paper [3] studies and analyses solution concepts for two-player normal form games with such preplay negotiation phase, under several assumptions for the bargaining power of the players, as well as the value of time for the players in such negotiations, and obtain results describing the possible solutions that can be achieved in such negotiation process in the resulting bargaining games. Strategic form games extended with preplay negotiations are further studied in [2], where where players can exchange unconditional offers, which substantially changes their strategic behavior. The preplay phase itself generates an unbounded-horizon extensive-form preplay negotiations game, in which the optimal strategic behavior of the players is analyzed and an algorithmic method for computing players' best offers is developed in [2], under the assumption of myopic rationality reasoning. 
Besides, somewhat more general types of contractual side payments-not only positive but also possibly negative (threats for punishments), and contingent not just on the recipient's strategy but also on their own actions, or on an entire strategy profile (i.e., on an outcome) - were studied before in the literature, most notably in [12,13], and later in the follow-up [14]. There are some essential differences in the assumptions made in those papers and in the present work, and respectively in the game-theoretic properties and effects of such payments, as demonstrated in [2,3], to which I refer the reader for further details on related work. As shown in the present paper, the game matrix transformations induced by preplay offers considered here can simulate negative payments, but not the type of offers for contractual payments contingent on strategy profiles considered in [12,14].

\subsection{Background}

The reader is expected to have basic background on N-person normal form games, e.g., $[9,10]$, as well as basic background on linear algebra within any standard textbook, e.g., [15], and on theory of groups, again within any standard reference on abstract algebra, e.g., [16].

\subsection{The Structure of the Paper}

Section 2 begins with an illustrating example, showing how the Prisoners Dilemma can be solved in a Pareto optimal way by players exchanging preplay offers, and then provides the basic definition related to preplay offers and payoff matrix transformations. Section 3 introduces the group of offer-induced game transformations for a given $\mathrm{N}$-player normal form game. Section 4 characterizes explicitly the class of normal form game payoff matrices that can be produced by means of such offer-induced game transformations from a given two-player game. Section 5 extends that characterization result to $\mathrm{N}$-player games and illustrates it with an example. The paper ends with the concluding Section 6.

\section{Preplay Offers and Payoff Matrix Transformations}

2.1. An Illustrating Example

Consider a standard version of the Prisoners' Dilemma (PD) game with a payoff matrix

\begin{tabular}{c|c|c}
$\mathrm{I} \backslash \mathrm{II}$ & $\mathrm{C}$ & $\mathrm{D}$ \\
\hline $\mathrm{C}$ & 4,4 & 0,5 \\
\hline $\mathrm{D}$ & 5,0 & 1,1
\end{tabular}

The only Nash Equilibrium is (D,D) with the paltry payoff $(1,1)$.

Now, suppose I makes to II a binding offer to pay her two utils after the game if II plays C. That offer transforms the game by transferring two utils from the payoff of I to the payoff of II in every entry of the column of the game matrix, where II plays C, as follows:

$$
\begin{array}{c|c|c}
\mathrm{I} \backslash \mathrm{II} & \mathrm{C} & \mathrm{D} \\
\hline \mathrm{C} & 2,6 & 0,5 \\
\hline \mathrm{D} & 3,2 & 1,1
\end{array}
$$

In this transformed game, player I still has the incentive to play $\mathrm{D}$, which strictly dominates $C$ for him, but the dominant strategy for II now is C; thus, the only Nash Equilibrium is $(D, C)$ with payoff $(3,2)$, which is strictly better than the original payoff $(1,1)$.

Of course, player II can now realize that player I has no incentive to cooperate yet. That incentive, however, can be created if player II makes a respective offer to pay two utils to player I after the game if player I cooperates. Then, the game transforms again, as follows:

\begin{tabular}{c|c|c}
$\mathrm{I} \backslash \mathrm{II}$ & $\mathrm{C}$ & $\mathrm{D}$ \\
\hline $\mathrm{C}$ & 4,4 & 2,3 \\
\hline $\mathrm{D}$ & 3,2 & 1,1
\end{tabular}


In this game, the only Nash Equilibrium is $(C, C)$ with payoff $(4,4)$, which is Pareto optimal. This is the same payoff for $(C, C)$ as in the original PD game, but now both players have transformed the game into one where they both have incentives to cooperate, and thus escape from the trap of the original Nash Equilibrium (D,D).

\subsection{Transformations of Normal Form Games by Preplay Offers}

Now, let us generalize. Consider a general two-person (for technical simplicity only) normal form (NF) game with a payoff matrix

\begin{tabular}{c|c|c|c|c}
$A \backslash B$ & $B_{1}$ & $\cdots$ & $B_{j}$ & $\cdots$ \\
\hline$A_{1}$ & $\cdots$ & $\cdots$ & $a_{1 j}, b_{1 j}$ & $\cdots$ \\
\hline$A_{2}$ & $\cdots$ & $\cdots$ & $a_{2 j}, b_{2 j}$ & $\cdots$ \\
\hline$\cdots$ & $\cdots$ & $\cdots$ & $\cdots$ & $\cdots$ \\
\hline$A_{i}$ & $\cdots$ & $\cdots$ & $a_{i j}, b_{i j}$ & $\cdots$ \\
\hline$\cdots$ & $\cdots$ & $\cdots$ & $\cdots$ & $\cdots$ \\
\hline
\end{tabular}

Suppose player A makes a preplay offer to player B to pay her additional utility $\delta \geq 0$ if $B$ plays $B_{j}$. I will denote such preplay offer by $A \stackrel{\delta / B_{j}}{\longrightarrow} B$.

(Of course, preplay offers where $\delta=0$ make no difference in the game. The technical reason for which we allow $\delta=0$ is to have an identity transformation at hand, but such vacuous offers can also be used by players for signalling.)

That offer transforms the payoff matrix of the game as follows:

\begin{tabular}{c|c|c|c|c}
$A \backslash B$ & $B_{1}$ & $\cdots$ & $B_{j}$ & $\cdots$ \\
\hline$A_{1}$ & $\cdots$ & $\cdots$ & $a_{1 j}-\delta, b_{1 j}+\delta$ & $\cdots$ \\
\hline$A_{2}$ & $\cdots$ & $\cdots$ & $a_{2 j}-\delta, b_{2 j}+\delta$ & $\cdots$ \\
\hline$\cdots$ & $\cdots$ & $\cdots$ & $\cdots$ & $\cdots$ \\
\hline$A_{i}$ & $\cdots$ & $\cdots$ & $a_{i j}-\delta, b_{i j}+\delta$ & $\cdots$ \\
\hline$\cdots$ & $\cdots$ & $\cdots$ & $\cdots$ & $\cdots$ \\
\hline
\end{tabular}

I will call such transformation of a payoff matrix a primitive offer-induced transformation, or a POI-transformation, for short.

Several preplay offers can be made by each player. Clearly, the transformation of a payoff matrix induced by several preplay offers can be obtained by applying the POItransformations corresponding to each of the offers consecutively, in any order. I will call such transformations general offer-induced transformations, or GOI-transformations, for short. Thus, every GOI-transformation corresponds to a finite set of preplay offers, and respectively, a set of POI-transformations. Note that the sets generating a given GOI-transformation need not be unique. For instance, A can make two independent consecutive offers

$A \stackrel{\delta_{1} / B_{j}}{\longrightarrow} B$ and $A \stackrel{\delta_{2} / B_{j}}{\longrightarrow} B$ equivalent to the offer $A \stackrel{\delta_{1}+\delta_{2} / B_{j}}{\longrightarrow} B$.

Thus, every GOI-transformation has a canonical form

$$
\left\{A \stackrel{\delta_{1}^{A} / B_{1}}{\longrightarrow} B, \ldots, A \stackrel{\delta_{m}^{A} / B_{m}}{\longrightarrow} B\right\} \cup\left\{B \stackrel{\delta_{1}^{B} / A_{1}}{\longrightarrow} A, \ldots, B \stackrel{\delta_{n}^{B} / A_{n}}{\longrightarrow} A\right\}
$$

for some non-negative numbers $\delta_{1}^{A}, \ldots \delta_{m}^{A}, \delta_{1}^{B}, \ldots \delta_{n}^{B}$.

That transformation changes the payoffs from $\left(a_{i j}, b_{i j}\right)$ to $\left(\hat{a}_{i j}, \hat{b}_{i j}\right)$ as follows:

$$
\hat{a}_{i j}=a_{i j}-\delta_{j}^{A}+\delta_{i}^{B}, \hat{b}_{i j}=b_{i j}+\delta_{j}^{A}-\delta_{i}^{B} .
$$

Note that the players can collude to make any outcome, with any non-negative redistribution of the payoffs in it, a strictly dominant strategy equilibrium by exchanging sufficiently high offers to make the strategies generating that outcome strictly dominant. 
Remark: clearly, rational players would only be interested in making offers inducing payoffs that are optimal for them. Thus, a 'preplay negotiation phase' emerges, which is studied in [1-3]. In this paper I do not take rationality considerations into account.

\section{The Group of Offer-Induced Game Transformations}

All definitions and observations in the previous section generalize in a straightforward way to $N$-person games, for any fixed integer $N>2$. I will name the $N$ players by $\{1,2, \ldots N\}$, but will still usually denote players by $A$ and $B$, possibly indexed. If an $N$ person game payoff matrix $M$ is such that for each $i \in\{1,2, \ldots N\}$ player $i$ has $m_{i}$ available strategies, we say that $M$ has dimension $\operatorname{dim}(M)=m_{1} \times m_{2} \times \ldots \times m_{N}$. Two game payoff matrices match if they have the same dimension.

I begin with some useful general observations on GOI-transformations of NF games for any fixed number of players $N$.

1. A GOI-transformation does not change the dimension of the game matrix, nor the sum of the payoffs of all players in any outcome in the matrix; it only redistributes them. In particular, GOI-transformations preserve the class of zero-sum games.

2. A GOI-transformation induced by a preplay offer of player A does not change the preferences of A regarding their own strategies. In particular, dominance between strategies of player $\mathrm{A}$ is invariant under GOI-transformations induced by preplay offers of A, i.e., a strategy $A_{i}$ dominates (weakly, respectively strongly) a strategy $A_{j}$ before a transformation induced by a preplay offer made by $\mathrm{A}$ if, and only if, $A_{i}$ dominates (weakly, respectively strongly) $A_{j}$ after the transformation.

Proposition 1. The set of all GOI-transformations of payoff matrices of $N$-person strategic games of any fixed dimension, for any given $N>1$ forms a commutative group under composition.

Proof. In this proof, we can restrict attention to the set of GOI-transformations of game matrices of a fixed given dimension. The composition of two such GOI-transformations is a GOI-transformation of the same type, corresponding to the union of the preplay offers generating the two transformations. Furthermore, the composition of GOI-transformations is clearly associative and commutative, because the order of transforming the matrix with regards to the primitive preplay offers generating the composed GOI-transformations is not essential.

The identity GOI-transformation is the one corresponding to any offer $A \stackrel{0 / B_{j}}{\longrightarrow} B$. Note that, even though there are many offers of this type, they generate the same transformation.

The existence of an inverse GOI-transformation to any GOI-transformation is a bit trickier, because an offered payment cannot be negative. However, note first that the inverse of the POI-transformation corresponding to an offer $A \stackrel{\delta / B_{j}}{\longrightarrow} B$ can be composed from the following offers:

- $\quad$ Player $A$ makes an offer $A \stackrel{\delta / B_{k}}{\longrightarrow} B$ for every strategy $B_{k}$ of $\mathrm{B}$, for $k \neq j$. That basically means that $\mathrm{A}$ offers a reward $\delta$ to $\mathrm{B}$ if $\mathrm{B}$ does not play the strategy $B_{j}$.

- $\quad$ Player B makes an offer $B \stackrel{\delta / A_{i}}{\longrightarrow} A$ for every strategy $A_{i}$ of $\mathrm{A}$. That basically means that $\mathrm{B}$ offers unconditionally a refund $\delta$ to $\mathrm{A}$.

The cumulative effect of these offers is that none of $A$ and $B$ gets anything from the other if B plays any $B_{k}$ where $k \neq j$, but if B plays $B_{j}$ then she effectively pays back an amount $\delta$ to $\mathrm{A}$, thus canceling the offer $A \stackrel{\delta / B_{j}}{\longrightarrow} B$

Finally, the inverse of any GOI-transformation $\mathcal{T}$ is obtained by composing the inverses of the primitive OI-transformations, of which $\mathcal{T}$ is composed.

The above proof also shows that offers for negative payments (i.e., threats of punishments) can be affected by GOI-transformations, too. Thus, henceforth we may assume that 
an offered payment may be any real number and consider the respectively extended class of GOI-transformations of the following canonical form:

$$
\left\{A \stackrel{\delta_{1}^{A} / B_{1}}{\longrightarrow} B, \ldots, A \stackrel{\delta_{m}^{A} / B_{m}}{\longrightarrow} B\right\} \cup\left\{B \stackrel{\delta_{1}^{B} / A_{1}}{\longrightarrow} A, \ldots, B \stackrel{\delta_{n}^{B} / A_{n}}{\longrightarrow} A\right\}
$$

for some real numbers $\delta_{1}^{A}, \ldots \delta_{m}^{A}, \delta_{1}^{B}, \ldots \delta_{n}^{B}$.

I note that GOI-transformations can also simulate more complex offers, such as the conditional offers considered in [3]. On the other hand, it will follow from Theorem 1 that GOI-transformations cannot simulate the contractual offers considered in [12,14], contingent not just on the recipient's strategy, but on an entire strategy profile (i.e., on an outcome).

I denote the group of GOI-transformations of $\mathrm{N}$-person NF games of a given dimension $D$ by $\mathcal{G}_{O I T}^{D}$. For a given payoff matrix $M$, let us denote by $\mathcal{G}_{\text {OIT }}^{D}(M)$ of dimension $D$ the orbit of $M$ under $\mathcal{G}_{\mathrm{O} I T}^{D}$, i.e., the result of the action of that group on $M$, which is the set of all payoff matrices obtained by applying GOI-transformations to $M$. I will also call $\mathcal{G}_{O I T}^{D}(M)$ the offer-induced transformation type (OIT type) of $M$. Since $\mathcal{G}_{O I T}^{D}$ is a group, the set of OIT types of NF game matrices forms a partition of the set of NF game matrices and thus generates an equivalence relation on that set.

\section{Characterizing the GOI-Transformations of 2-Person NF Games}

I leave the general algebraic theory of GOI-transformations and the groups $\mathcal{G}_{\mathrm{OIT}}^{\mathrm{D}}$ to further study, and here I address the question: given a game payoff matrix $M$, how is the OIT type $\mathcal{G}_{\mathrm{OIT}}^{D}(M)$ of $M$ characterized and constructed? That is, what are the possible results of GOI-transformations of $\mathcal{M}$ ?

Let us first rephrase the question: given another game matrix $\widehat{M}$ of the same dimension, can $\widehat{M}$ be obtained from $M$ by a GOI-transformation?

To answer this question, let us first consider, for technical simplicity, the case of twoperson games. Let

$M=$\begin{tabular}{c|c|c|c|c|c|}
$A \backslash B$ & $B_{1}$ & $\cdots$ & $B_{j}$ & $\cdots$ & $B_{m}$ \\
\hline$A_{1}$ & $a_{11}, b_{11}$ & $\cdots$ & $a_{1 j}, b_{1 j}$ & $\cdots$ & $a_{1 m}, b_{1 m}$ \\
\hline$\cdots$ & $\cdots$ & $\cdots$ & $\cdots$ & $\cdots$ & $\cdots$ \\
\hline$A_{i}$ & $a_{i 1}, b_{i 1}$ & $\cdots$ & $a_{i j}, b_{i j}$ & $\cdots$ & $a_{i m}, b_{i m}$ \\
\hline$\cdots$ & $\cdots$ & $\cdots$ & $\cdots$ & $\cdots$ & $\cdots$ \\
\hline$A_{n}$ & $a_{n 1}, b_{n 1}$ & $\cdots$ & $a_{n j}, b_{n j}$ & $\cdots$ & $a_{n m}, b_{n m}$ \\
\hline
\end{tabular}

Further I will use a compact notation for $M$ as follows: $M=\left[a_{i j}, b_{i j}\right]_{\substack{i=1, \ldots, n \\ j=1, \ldots, m}}$.

Now, let

$\widehat{M}=$\begin{tabular}{c|c|l|c|l|c|}
$A \backslash B$ & $B_{1}$ & $\cdots$ & $B_{j}$ & $\cdots$ & $B_{m}$ \\
\hline$A_{1}$ & $\hat{a}_{11}, \hat{b}_{11}$ & $\cdots$ & $\hat{a}_{1 j}, \hat{b}_{1 j}$ & $\cdots$ & $\hat{a}_{1 m}, \hat{b}_{1 m}$ \\
\hline$\cdots$ & $\cdots$ & $\cdots$ & $\cdots$ & $\cdots$ & $\cdots$ \\
\hline$A_{i}$ & $\hat{a}_{i 1}, \hat{b}_{i 1}$ & $\cdots$ & $\hat{a}_{i j}, \hat{b}_{i j}$ & $\cdots$ & $\hat{a}_{i m}, \hat{b}_{i m}$ \\
\hline$\cdots$ & $\cdots$ & $\cdots$ & $\cdots$ & $\cdots$ & $\cdots$ \\
\hline$A_{n}$ & $\hat{a}_{n 1}, \hat{b}_{n 1}$ & $\cdots$ & $\hat{a}_{n j}, \hat{b}_{n j}$ & $\cdots$ & $\hat{a}_{n m}, \hat{b}_{n m}$ \\
\hline
\end{tabular}

Suppose $\widehat{M}$ can be obtained from $M$ by a GOI-transformation

$$
\tau=\left\{A \stackrel{\delta_{1}^{A} / B_{1}}{\longrightarrow} B, \ldots, A \stackrel{\delta_{m}^{A} / B_{m}}{\longrightarrow} B\right\} \cup\left\{B \stackrel{\delta_{1}^{B} / A_{1}}{\longrightarrow} A, \ldots, B \stackrel{\delta_{n}^{B} / A_{n}}{\longrightarrow} A\right\}
$$

for some real numbers $\delta_{1}^{A}, \ldots \delta_{m}^{A}, \delta_{1}^{B}, \ldots \delta_{n}^{B}$.

Recall, that the transformation $\tau$ changes the payoffs as follows:

$$
\hat{a}_{i j}=a_{i j}+\delta_{i}^{B}-\delta_{j}^{A}, \quad \hat{b}_{i j}=b_{i j}+\delta_{j}^{A}-\delta_{i}^{B} .
$$


Theorem 1. Let $M=\left[a_{i j}, b_{i j}\right]_{\substack{i=1, \ldots, n \\ j=1, \ldots, m}}$ and $\widehat{M}=\left[\hat{a}_{i j}, \hat{b}_{i j}\right]_{\substack{i=1, \ldots, n \\ j=1, \ldots, m}}$ be 2-person NF game matrices of dimension $n \times m$. The matrix $\widehat{M}$ can be obtained from $M$ by a GOI-transformation if and only if the following two conditions hold, where $c_{i j}=\hat{a}_{i j}-a_{i j}$ and $d_{i j}=\hat{b}_{i j}-b_{i j}$ :

$C_{1}: \quad a_{i j}+b_{i j}=\hat{a}_{i j}+\hat{b}_{i j}$, or equivalently, $d_{i j}=-c_{i j}$, for all $i=1, \ldots n, j=1, \ldots m$.

$c_{2}: \quad c_{i j}-c_{i(j+1)}=c_{(i+1) j}-c_{(i+1)(j+1)}$, or equivalently, $c_{i j}+c_{(i+1)(j+1)}=c_{i(j+1)}+c_{(i+1) j}$, for all $i=1, \ldots n-1, j=1, \ldots m-1$.

Proof. Condition $C_{1}$ is obviously necessary, because all side payments are between the two players, so the sum of their payoffs in any given outcome remains constant.

To show the necessity of $C_{2}$, a simple calculation suffices:

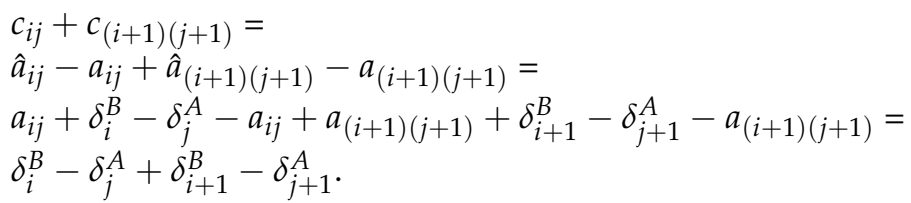

Likewise:

$c_{i(j+1)}+c_{(i+1) j}=$

$\hat{a}_{i(j+1)}-a_{i(j+1)}+\hat{a}_{(i+1) j}-a_{(i+1) j}=$

$a_{i(j+1)}+\delta_{i}^{B}-\delta_{j+1}^{A}-a_{i(j+1)}+a_{(i+1) j}+\delta_{i+1}^{B}-\delta_{j}^{A}-a_{(i+1) j}=$

$\delta_{i}^{B}-\delta_{j+1}^{A}+\delta_{i+1}^{B}-\delta_{j}^{A}$.

Clearly, the two results are equal.

For the sufficiency, suppose $C_{1}$ and $C_{2}$ hold. Then, first observe that, due to $C_{1}$, any OI-transformation that transforms A's payoffs in $M$ into A's payoffs in $\widehat{M}$ will transform accordingly the payoffs of $\mathrm{B}$ in $M$ into the payoffs of $\mathrm{B}$ in $\widehat{M}$. So, we can ignore B's payoffs and consider only the transformation of the matrices of A's payoffs.

To prove the existence of (real valued) payments $\delta_{1}^{A}, \ldots \delta_{m}^{A}, \delta_{1}^{B}, \ldots \delta_{n}^{B}$ that effect the transformation from $M$ to $\widehat{M}$, we consider the system (indicated below with " $\|$ ") of $m n$ linear equations for these $n+m$ unknowns that expresses the changes of A's payoffs:

$$
\| \delta_{i}^{B}-\delta_{j}^{A}=c_{i j}, \text { for all } i=1, \ldots n, j=1, \ldots m .
$$

Thus, $\widehat{M}$ can be obtained from $M$ by a GOI-transformation precisely when that system has a real solution. The rest is a simple application of standard linear algebra. The matrix of the system is

$$
\left(\begin{array}{cccccccc|c}
\delta_{1}^{B} & \delta_{2}^{B} & \ldots & \delta_{n}^{B} & \delta_{1}^{A} & \delta_{2}^{A} & \ldots & \delta_{m}^{A} & c_{i j} \\
\hline 1 & 0 & 0 & 0 & -1 & 0 & 0 & 0 & c_{11} \\
1 & 0 & 0 & 0 & 0 & -1 & 0 & 0 & c_{12} \\
\ldots & \ldots & \ldots & \ldots & \ldots & \ldots & \ldots & \ldots & \ldots \\
1 & 0 & 0 & 0 & 0 & 0 & 0 & -1 & c_{1 m} \\
0 & 1 & 0 & 0 & -1 & 0 & 0 & 0 & c_{21} \\
0 & 1 & 0 & 0 & 0 & -1 & 0 & 0 & c_{22} \\
\ldots & \ldots & \ldots & \ldots & \ldots & \ldots & \ldots & \ldots & \ldots \\
0 & 1 & 0 & 0 & 0 & 0 & 0 & -1 & c_{2 m} \\
\ldots & \ldots & \ldots & \ldots & \ldots & \ldots & \ldots & \ldots & \ldots \\
\ldots & \ldots & \ldots & \ldots & \ldots & \ldots & \ldots & \ldots & \ldots \\
0 & 0 & 0 & 1 & -1 & 0 & 0 & 0 & c_{n 1} \\
0 & 0 & 0 & 1 & 0 & -1 & 0 & 0 & c_{n 2} \\
\ldots & \ldots & \ldots & \ldots & \ldots & \ldots & \ldots & \ldots & \ldots \\
0 & 0 & 0 & 1 & 0 & 0 & 0 & -1 & c_{n m}
\end{array}\right)
$$


We now apply to it the standard Gauss-Jordan elimination method. Subtracting row 1 from each of rows $2, \ldots, m$, then row $m+1$ from rows $m+2, \ldots, 2 m$, etc., and finally row $(n-1) m+1$ from rows $(n-1) m+2, \ldots, n m$ produces:

$$
\left(\begin{array}{cccccccc|c}
\delta_{1}^{B} & \delta_{2}^{B} & \ldots & \delta_{n}^{B} & \delta_{1}^{A} & \delta_{2}^{A} & \ldots & \delta_{m}^{A} & c_{i j} \\
\hline 1 & 0 & 0 & 0 & -1 & 0 & 0 & 0 & c_{11} \\
0 & 0 & 0 & 0 & 1 & -1 & 0 & 0 & c_{12}-c_{11} \\
\ldots & \ldots & \ldots & \ldots & \ldots & \ldots & \ldots & \ldots & \ldots \\
0 & 0 & 0 & 0 & 1 & 0 & 0 & -1 & c_{1 m}-c_{11} \\
0 & 1 & 0 & 0 & -1 & 0 & 0 & 0 & c_{21} \\
0 & 0 & 0 & 0 & 1 & -1 & 0 & 0 & c_{22}-c_{21} \\
\ldots & \ldots & \ldots & \ldots & \ldots & \ldots & \ldots & \ldots & \ldots \\
0 & 0 & 0 & 0 & 1 & 0 & 0 & -1 & c_{2 m}-c_{21} \\
\ldots & \ldots & \ldots & \ldots & \ldots & \ldots & \ldots & \ldots & \ldots \\
\ldots & \ldots & \ldots & \ldots & \ldots & \ldots & \ldots & \ldots & \ldots \\
0 & 0 & 0 & 1 & -1 & 0 & 0 & 0 & c_{n 1} \\
0 & 0 & 0 & 1 & 0 & -1 & 0 & 0 & c_{n 2}-c_{n 1} \\
\ldots & \ldots & \ldots & \ldots & \ldots & \ldots & \ldots & \ldots & \ldots \\
0 & 0 & 0 & 1 & 0 & 0 & 0 & -1 & c_{n m}-c_{n 1}
\end{array}\right)
$$

Now, note that rows $k, m+k, \ldots(n-1) m+k$ have the same left-hand sides, for each $k=2,3, \ldots, m$. For the system to be consistent, the right-hand sides must be equal, too. Indeed, by consecutive applications of condition $C_{2}: c_{1 k}-c_{11}=c_{2 k}-c_{21}=\ldots c_{n k}-c_{n 1}$.

Further, we subtract row $k$ from each of $m+k, \ldots(n-1) m+k$, for each $k=2,3, \ldots, m$ and remove the resulting 0 -rows. After rearrangement of the remaining rows we obtain:

$$
\left(\begin{array}{cccccccc|c}
\delta_{1}^{B} & \delta_{2}^{B} & \ldots & \delta_{n}^{B} & \delta_{1}^{A} & \delta_{2}^{A} & \ldots & \delta_{m}^{A} & c_{i j} \\
\hline 1 & 0 & 0 & 0 & -1 & 0 & 0 & 0 & c_{11} \\
0 & 1 & 0 & 0 & -1 & 0 & 0 & 0 & c_{21} \\
\ldots & \ldots & \ldots & \ldots & \ldots & \ldots & \ldots & \ldots & \ldots \\
0 & 0 & 0 & 1 & -1 & 0 & 0 & 0 & c_{n 1} \\
0 & 0 & 0 & 0 & 1 & -1 & 0 & 0 & c_{12}-c_{11} \\
\ldots & \ldots & \ldots & \ldots & \ldots & \ldots & \ldots & \ldots & \ldots \\
0 & 0 & 0 & 0 & 1 & 0 & 0 & -1 & c_{1 m}-c_{11}
\end{array}\right)
$$

Finally, subtracting row $n+m-2$ from $n+m-1$, then row $n+m-3$ from $n+m-2$, etc., and lastly, row $n+1$ from $n+2$, we obtain:

$$
\left(\begin{array}{cccccccc|c}
\delta_{1}^{B} & \delta_{2}^{B} & \ldots & \delta_{n}^{B} & \delta_{1}^{A} & \delta_{2}^{A} & \ldots & \delta_{m}^{A} & c_{i j} \\
\hline 1 & 0 & 0 & 0 & -1 & 0 & 0 & 0 & c_{11} \\
0 & 1 & 0 & 0 & -1 & 0 & 0 & 0 & c_{21} \\
\ldots & \ldots & \ldots & \ldots & \ldots & \ldots & \ldots & \ldots & \ldots \\
0 & 0 & 0 & 1 & -1 & 0 & 0 & 0 & c_{n 1} \\
0 & 0 & 0 & 0 & 1 & -1 & 0 & 0 & c_{12}-c_{11} \\
0 & 0 & 0 & 0 & 0 & 1 & -1 & 0 & c_{13}-c_{12} \\
\ldots & \ldots & \ldots & \ldots & \ldots & \ldots & \ldots & \ldots & \ldots \\
0 & 0 & 0 & 0 & 0 & 0 & 1 & -1 & c_{1 m}-c_{1(m-1)}
\end{array}\right)
$$

The ranks of the matrix and the extended matrix above are clearly equal to $n+m-1$, so the corresponding system is consistent. Moreover, it has infinitely many solutions obtained by treating $\delta_{m}^{A}$ as a real parameter and solving for all other unknowns in terms of it. This completes the proof.

Note that if either of the conditions $\mathrm{C} 1$ and $\mathrm{C} 2$ of Theorem 1 holds for payoff matrices $M$ and $\widehat{M}$, then it respectively holds for $M^{\prime}$ and $\widehat{M^{\prime}}$, where $M^{\prime}$ is a payoff matrix obtained by permuting the order of strategies of $M$. 
Corollary 1 . In the case of $2 \times 2$ payoff matrices

$$
M=\left[\begin{array}{c|cc}
A \backslash B & B_{1} & B_{2} \\
\hline A_{1} & a_{11}, b_{11} & a_{12}, b_{12} \\
A_{2} & a_{21}, b_{21} & a_{22}, b_{22}
\end{array}\right], \widehat{M}=\left[\begin{array}{c|cc}
A \backslash B & B_{1} & B_{2} \\
\hline A_{1} & \hat{a}_{11}, \hat{b}_{11} & \hat{a}_{12}, \hat{b}_{12} \\
A_{2} & \hat{a}_{21}, \hat{b}_{21} & \hat{a}_{22}, \hat{b}_{22}
\end{array}\right]
$$

the matrix $\widehat{M}$ can be obtained from $M$ by a GOI-transformation if and only if:

$C_{1}: a_{i j}+b_{i j}=\hat{a}_{i j}+\hat{b}_{i j}$, for all $i, j \in\{1,2\}$.

$c_{2}: \quad c_{11}+c_{22}=c_{12}+c_{21}$, where $c_{i j}=\hat{a}_{i j}-a_{i j}$.

Corollary 2. No OI-transformation applied to a game matrix $M$ can produce a game matrix that differs from $M$ in only one outcome.

Consequently, OI-transformations cannot simulate the offers considered in [12,14], contingent on a single outcome.

Example 1. For example, the payoff matrix

$\left[\begin{array}{c|cc}A \backslash B & B_{1} & B_{2} \\ \hline A_{1} & 4,4 & 0,5 \\ A_{2} & 3,0 & 1,1\end{array}\right]$

can be OI-transformed to

$$
\left[\begin{array}{c|cc}
A \backslash B & B_{1} & B_{2} \\
\hline A_{1} & 2,6 & 2,3 \\
A_{2} & 0,3 & 2,0
\end{array}\right]
$$

but not to

$$
\left[\begin{array}{c|cc}
A \backslash B & B_{1} & B_{2} \\
\hline A_{1} & 2,6 & 2,3 \\
A_{2} & 0,3 & 1,1
\end{array}\right]
$$

neither to

$$
\left[\begin{array}{c|cc}
A \backslash B & B_{1} & B_{2} \\
\hline A_{1} & 2,6 & 3,2 \\
A_{2} & 0,3 & 2,0
\end{array}\right]
$$

The condition $C_{2}$ from Theorem 1 can be rewritten as a recurrent formula $c_{(i+1)(j+1)}=$ $c_{(i+1) j}+c_{i(j+1)}-c_{i j}$, which suggests that all values of $c_{i j}$, and therefore all values of $\hat{a}_{i j}$, can be computed iteratively from some initial values along one row and one column. Therefore, by using conditions $C_{1}$ and $C_{2}$, every OI-transformation can be determined locally, by specifying the resulting payoffs for any of the two players on one row and one column. In other words, fix a strategy profile in the transformed matrix, fix one of the players, and assign any real payoffs for that player to all outcomes where at least one of the players follows their strategy from the fixed strategy profile. The resulting partly defined matrix can then be uniquely extended to one that can be obtained by an OI-transformation from the initial matrix $M$. The following result formalizes this observation.

Theorem 2. Let $M=\left[a_{i j}, b_{i j}\right]_{\substack{i=1, \ldots, n \\ j=1, \ldots, m}}$. Then for every fixed $i \in\{1, \ldots, n\}$ and $j \in\{1, \ldots, m\}$, every tuple of $n+m-1$ reals $\hat{a}_{i 1}, \ldots, \hat{a}_{i m}, \hat{a}_{1 j}, \ldots, \hat{a}_{n j}$ can be extended to a unique payoff matrix $\widehat{M}=\left[\hat{a}_{i j}, \hat{b}_{i j}\right]_{\substack{i=1, \ldots, n \\ j=1, \ldots, m}}$ that can be obtained from $M$ by a GOI-transformation. 
Proof. For notational simplicity, let us assume that $i=1$ and $j=1$ and that $m \leq n$. Clearly, the argument for any other combination of $i, j$ is analogous. To determine the matrix $\widehat{M}$ it suffices to determine the values of all $\hat{a}_{i j}$, for $i=1, \ldots, n, j=1, \ldots, m$ and then compute all values $\hat{b}_{i j}$ by applying condition $C_{1}$ from Theorem 1 .

Now, note that all values $\hat{a}_{i j}$ can be computed iteratively, step-by-step, by using the identities in $C_{2}$ of Theorem 1: first, compute $\hat{a}_{22}$; then, $\hat{a}_{23}$ and $\hat{a}_{32}$, etc.. More precisely, given all values along the diagonal $\hat{a}_{1 k}, \hat{a}_{2(k-1)} \ldots, \hat{a}_{k 1}$, for $k<n$, using $C_{2}$ of Theorem 1 one can compute uniquely values for $\hat{a}_{2(k+1)}, \hat{a}_{2 k} \ldots, \hat{a}_{(k+1) 2}$. When $k$ increases between $m$ and $n$, the argument continues likewise, but for values along the diagonals $\hat{a}_{k m}, \ldots, \hat{a}_{2(m+k-2)}$, and then further, along the shrinking diagonals $\hat{a}_{k m}, \ldots, \hat{a}_{j(m+k-j)}$, until eventually $\hat{a}_{n m}$ is computed.

The resulting matrix $\widehat{M}=\left[\hat{a}_{i j}, \hat{b}_{i j}\right]_{\substack{i=1, \ldots, n \\ j=1, \ldots, m}}$ satisfies the conditions $C_{1}$ and $C_{2}$ by construction. Therefore, by Theorem $1, \widehat{M}$ can be obtained from $M$ by a GOI-transformation. The uniqueness of $\widehat{M}$ follows from the construction, too.

The case of arbitrary $i$ and $j$ is essentially the same, but the computation of the values of the $\hat{a}_{p q} \mathrm{~s}$ now propagates from $\hat{a}_{i j}$ in all four diagonal directions.

Thus, in summary, Theorems 1 and 2 together say that any payoff matrix $\widehat{M}$ can be obtained from matrix $M$ by a GOI-transformation by choosing suitable payoffs in one row and one column satisfying condition $C_{1}$, and then computing the rest by using the recurrent formulae derived from condition $C_{2}$. In particular, that means that for any fixed strategy profile of the two players in a given NF-game with matrix $M$ and respective desired outcomes corresponding to each of the strategies in the profile, which are consistent with the conditions $M$ (i.e., preserving the total payoff in each outcome) there is a unique GOI-transformation of $M$ producing a payoff matrix with the desired outcomes.

Example 2. Suppose the starting payoff matrix is

$$
M=\left[\begin{array}{c|ccc}
A \backslash B & B_{1} & B_{2} & B_{3} \\
\hline A_{1} & 4,4 & 6,2 & 0,6 \\
A_{2} & 2,6 & 1,1 & 2,2 \\
A_{3} & 5,0 & 0,1 & 1,5 \\
A_{4} & 0,0 & 2,3 & 3,0
\end{array}\right]
$$

and row 1 and column 1 of the transformed matrix are as follows:

$$
\widehat{M}=\left[\begin{array}{c|ccc}
A \backslash B & B_{1} & B_{2} & B_{3} \\
\hline A_{1} & 1,7 & 4,4 & 2,4 \\
A_{2} & 7,1 & & \\
A_{3} & 3,2 & & \\
A_{4} & 0,0 & &
\end{array}\right]
$$

The remaining entries are computed consecutively from condition $C_{2}$ of Theorem 1:

First, we obtain $c_{22}=c_{21}+c_{12}-c_{11}=5+(-2)-(-3)=6$. -1 , etc.

Then: $c_{23}=c_{22}+c_{13}-c_{12}=6+2-(-2)=10 ; c_{32}=c_{31}+c_{22}-c_{21}=-2+6-5=$ 
Thus, the whole matrix $C=\left(c_{i j}\right)_{\substack{i=1, \ldots, 4 \\ j=1, \ldots, 3}}$ of differences $c_{i j}$ (representing the side payments to be received by player $A$ ) is computed as follows:

$$
C=\left[\begin{array}{c|rrr}
i \backslash j & 1 & 2 & 3 \\
\hline 1 & -3 & -2 & 2 \\
2 & 5 & 6 & 10 \\
3 & -2 & -1 & 3 \\
4 & 0 & 1 & 5
\end{array}\right]
$$

Eventually, from the definition of $c_{i j}$ and Condition $C_{1}$ of Theorem 1, we obtain:

$$
\widehat{M}=\left[\begin{array}{c|ccc}
A \backslash B & B_{1} & B_{2} & B_{3} \\
\hline A_{1} & 1,7 & 4,4 & 2,4 \\
A_{2} & 7,1 & 7,-5 & 12,-8 \\
A_{3} & 3,2 & -1,2 & 4,2 \\
A_{4} & 0,0 & 3,2 & 8,-5
\end{array}\right]
$$

\section{Characterizing the OI-Transformations of $N$-Person Normal Form Games}

Generalizing these results to $N$-person NF games is relatively easy, but it adds a substantial notational overhead.

Let the players be indexed with $\{1,2, \ldots N\}$ and consider two NF game matrices of the same dimension: $m_{1} \times m_{2} \times \ldots \times m_{N}$ :

$$
M=\left(\left\langle a_{i_{1} i_{2} \ldots i_{N}}^{1}, a_{i_{1} i_{2} \ldots i_{N}}^{2}, \ldots a_{i_{1} i_{2} \ldots i_{N}}^{N}\right\rangle\right) \underset{\substack{i_{k} \leq m_{k} \\ k=1,2, \ldots N}}{\substack{k \\ k=1}}
$$

and

$$
\begin{aligned}
& \widehat{M}\left.=\left(\left\langle\hat{a}_{i_{1} i_{2} \ldots i_{N}}^{1}, \hat{a}_{i_{1} i_{2} \ldots i_{N}}^{2}, \ldots \hat{a}_{i_{1} i_{2} \ldots i_{N}}^{N}\right\rangle\right)\right)_{\substack{i_{k} \leq m_{k} \\
k=1,2, \ldots N}} \\
& \text { Let } c_{i_{1} i_{2} \ldots i_{N}}^{k}=\hat{a}_{i_{1} i_{2} \ldots i_{N}}^{k}-a_{i_{1} i_{2} \ldots i_{N}}^{k} .
\end{aligned}
$$

Theorem 3. Let $M, \widehat{M}$ be $N$-person NF game matrices of dimension $m_{1} \times m_{2} \times \ldots \times m_{N}$. The matrix $\widehat{M}$ can be obtained from $M$ by a GOI-transformation if and only if the following two conditions hold:

$C_{1}^{N}: \quad \sum_{j=1}^{N} \hat{a}_{i_{1} i_{2} \ldots i_{N}}^{j}=\sum_{j=1}^{N} a_{i_{1} i_{2} \ldots i_{N}}^{j}$ for any $i_{k} \leq m_{k}, k=1,2, \ldots N$.

(The sum of all payoffs in any given outcome must remain the same.)

$C_{2}^{N}$ : For any fixed $k, j=1,2, \ldots N$ the difference

$$
d_{i_{1} \ldots i_{N}}^{j, k}:=c_{i_{1} \ldots i_{k}+1 \ldots i_{N}}^{j}-c_{i_{1} \ldots i_{k} \ldots i_{N}}^{j}
$$

is the same for every $i_{1}, \ldots, i_{j}, \ldots, i_{N}$ such that $i_{p} \leq m_{p}, p=1, \ldots, N$ and $i_{j}<m_{j}$.

Proof. The proofs follows the same reasoning as in the two-person case.

The necessity of condition $C_{1}^{N}$ is obvious. To show the necessity of condition $C_{2}^{N}$, we do again simple calculations. Let $\widehat{M}$ be obtained from $M$ by a GOI-transformation effected by side payment offers $\left\{\delta_{j}^{i, k}\right\}_{k=1, \ldots, N ; 1 \leq j \leq m_{j}}$, where $\delta_{j}^{i, k}$ is the side payment offered by player $i$ to player $k \neq i$ contingent on $k$ playing action $j$. For technical convenience I also put $\delta_{j}^{i, i}=0$ for any $j$. Then we have:

$$
c_{i_{1} \ldots i_{j} \ldots i_{N}}^{j}=\hat{a}_{i_{1} \ldots i_{j} \ldots i_{N}}^{j}-a_{i_{1} \ldots i_{j} \ldots i_{N}}^{j}=\sum_{i=1}^{N} \delta_{i_{j}}^{i, j}-\sum_{k=1}^{N} \delta_{i_{k}}^{j, k} .
$$


Note that it suffices to show that

$$
d_{i_{1} \ldots i_{p} \ldots i_{N}}^{j, k}=d_{i_{1} \ldots i_{p}+1 \ldots i_{N}}^{j, k}
$$

for any $j, k, p=1, \ldots, N$, i.e.

$$
c_{i_{1} \ldots i_{k}+1 \ldots i_{p} \ldots i_{N}}^{j}-c_{i_{1} \ldots i_{k} \ldots i_{p} \ldots i_{N}}^{j}=c_{i_{1} \ldots i_{k}+1 \ldots i_{p}+1 \ldots i_{N}}^{j}-c_{i_{1} \ldots i_{k} \ldots i_{p}+1 \ldots i_{N}}^{j}
$$

for $k<p \leq N$ and likewise

$$
c_{i_{1} \ldots i_{p} \ldots i_{k}+1 \ldots i_{N}}^{j}-c_{i_{1} \ldots i_{p} \ldots i_{k} \ldots i_{N}}^{j}=c_{i_{1} \ldots i_{p}+1 \ldots i_{k}+1 \ldots i_{N}}^{j}-c_{i_{1} \ldots i_{p}+1 \ldots i_{k} \ldots i_{N}}^{j}
$$

for $1 \leq p<k$. Both cases are completely analogous, so let us check the first equality. It is equivalent to

$$
c_{i_{1} \ldots i_{k}+1 \ldots i_{p} \ldots i_{N}}^{j}+c_{i_{1} \ldots i_{k} \ldots i_{p}+1 \ldots i_{N}}^{j}=c_{i_{1} \ldots i_{k}+1 \ldots i_{p}+1 \ldots i_{N}}^{j}+c_{i_{1} \ldots i_{k} \ldots i_{p} \ldots i_{N}}^{j}
$$

First, suppose $j \neq p, k$. By definition, we have for the left-hand side:

$c_{i_{1} \ldots i_{k}+1 \ldots i_{p} \ldots i_{N}}^{j}+c_{i_{1} \ldots i_{k} \ldots i_{p}+1 \ldots i_{N}}^{j}=$

$\sum_{q=1}^{N} \delta_{i_{j}}^{q, j}-\sum_{\substack{q=1 \\ q \neq k}}^{N} \delta_{i_{q}}^{j, q}-\delta_{i_{k}+1}^{j, k}+\sum_{q=1}^{N} \delta_{i_{j}}^{q, j}-\sum_{\substack{q=1 \\ q \neq p}}^{N} \delta_{i_{q}}^{j, q}-\delta_{i_{p}+1}^{j, p}$.

Respectively, for the right hand side:

$c_{i_{1} \ldots i_{k}+1 \ldots i_{p}+1 \ldots i_{N}}^{j}+c_{i_{1} \ldots i_{k} \ldots i_{p} \ldots i_{N}}^{j}=$

$\sum_{q=1}^{N} \delta_{i_{j}}^{q, j}-\sum_{\substack{q=1 \\ q \neq k, p}}^{N} \delta_{i_{q}}^{j, q}-\delta_{i_{p}+1}^{j, p}-\delta_{i_{k}+1}^{j, k}+\sum_{q=1}^{N} \delta_{i_{j}}^{q, j}-\sum_{q=1}^{N} \delta_{i_{q}}^{j, q}$.

A direct inspection shows that these are equal.

Now, consider the case where $j=p$. For the left hand side we get:

$c_{i_{1} \ldots i_{k}+1 \ldots i_{j} \ldots i_{N}}^{j}+c_{i_{1} \ldots i_{k} \ldots i_{j}+1 \ldots i_{N}}^{j}=$

$\sum_{q=1}^{N} \delta_{i_{j}}^{q, j}-\sum_{\substack{q=1 \\ q \neq k}}^{N} \delta_{i_{q}}^{j, q}-\delta_{i_{k}+1}^{j, k}+\sum_{q=1}^{N} \delta_{i_{j}+1}^{q, j}-\sum_{\substack{q=1 \\ q \neq j}}^{N} \delta_{i_{q}}^{j, q}-\delta_{i_{j}+1}^{j, j}$.

Respectively, for the right-hand side:

$c_{i_{1} \ldots i_{k}+1 \ldots i_{j}+1 \ldots i_{N}}^{j}+c_{i_{1} \ldots i_{k} \ldots i_{j} \ldots i_{N}}^{j}=$

$\sum_{q=1}^{N} \delta_{i_{j}+1}^{q, j}-\sum_{\substack{q=1 \\ q \neq k, j}}^{N} \delta_{i_{q}}^{j, q}-\delta_{i_{j}+1}^{j, j}-\delta_{i_{k}+1}^{j, k}+\sum_{q=1}^{N} \delta_{i_{j}}^{q, j}-\sum_{q=1}^{N} \delta_{i_{q}}^{j, q}$.

Again, by direct inspection we see that these are equal.

Finally, the case where $j=k$ is completely analogous.

Now, suppose conditions $C_{1}^{N}$ and $C_{2}^{N}$ hold for the matrices $M$ and $\widehat{M}$, and all parameters $c_{i_{1} \ldots i_{j} \ldots i_{N}}^{j}$ are defined as before. As in the two-person case, we can show that the system of equations

$$
\| \sum_{i=1}^{N} \delta_{i_{j}}^{i, j}-\sum_{k=1}^{N} \delta_{i_{k}}^{j, k}=c_{i_{1} \ldots i_{j} \ldots i_{N}}^{j}
$$

for all $j, i_{1}, \ldots, i_{j}, \ldots, i_{N}$ such that $i_{p} \leq m_{p}, p=1, \ldots, N$, for the unknown real payments $\left\{\delta_{j}^{i, k}\right\}_{k=1, \ldots, N ; 1 \leq j \leq m_{j}}$, is consistent. We can use again standard linear algebra and show that elementary matrix transformations would reduce the system to a consistent one in a canonical form. I omit the routine, though messy technicalities. 
As in the two-person case, every OI-transformation can be determined by the payoffs for all players in the outcomes along the rows in all coordinate directions passing from any fixed outcome, that is, all outcomes resulting from all but one players following a fixed strategy profile. Here is the formal result.

Theorem 4. Let

$$
M=\left(\left\langle a_{i_{1} i_{2} \ldots i_{N}}^{1}, a_{i_{1} i_{2} \ldots i_{N}}^{2}, \ldots a_{i_{1} i_{2} \ldots i_{N}}^{N}\right\rangle\right) \underset{\substack{i_{k} \leq m_{k} \\ k=1,2, \ldots N}}{\substack{k \\ k=1}}
$$

and $\left\langle p_{1} \ldots p_{N}\right\rangle$ be a fixed tuple such that $p_{k} \in\left\{1, \ldots, m_{k}\right\}$ for each $k=1, \ldots$. Then every tuple $\mathcal{T}=\left\langle\left\langle\hat{a}_{1 p_{2} \ldots p_{N}}^{k}, \hat{a}_{2 p_{2} \ldots p_{N}}^{k}, \ldots, \hat{a}_{m_{1} p_{2} \ldots p_{N}}^{k}, \hat{a}_{p_{1} 1 \ldots i_{N}}^{k}, \hat{a}_{p_{1} 2 \ldots i_{N}}^{k}, \ldots, \hat{a}_{p_{1} m_{2} \ldots p_{N}}^{k}, \ldots\right.\right.$, $\left.\hat{a}_{p_{1} p_{2} \ldots 1}^{k}, \hat{a}_{p_{1} p_{2} \ldots 2}^{k}, \ldots, \hat{a}_{p_{1} p_{2} \ldots m_{N}}^{k}\right\rangle|k=1, \ldots, N\rangle$

satisfying condition $C_{1}^{N}$ of Theorem 3 can be extended to a unique payoff matrix

$$
\widehat{M}=\left(\left\langle\hat{a}_{i_{1} i_{2} \ldots i_{N}}^{1}, \hat{a}_{i_{1} i_{2} \ldots i_{N}}^{2} \ldots \hat{a}_{i_{1} i_{2} \ldots i_{N}}^{N}\right\rangle\right)_{\substack{i_{k} \leq m_{k} \\ k=1,2, \ldots N}}
$$

that can be obtained from $M$ by a GOI-transformation.

Proof. Given any tuple of values $\mathcal{T}$ satisfying the conditions of the theorem, the extension to a matrix $\widehat{M}$ can be performed as follows. First, we use $\mathcal{T}$ and the entries of $M$ to compute the values of all $c_{i_{1} \ldots i_{N}}^{j}$ whose index vectors corresponding to the entries in $\mathcal{T}$.

We take the identities

$$
d_{i_{1} \ldots i_{p} \ldots i_{N}}^{j, k}=d_{i_{1} \ldots i_{p}+1 \ldots i_{N}}^{j, k} \text { for all } j, k, p=1, \ldots, N
$$

and expand them (assuming e.g., that $k<p \leq N$ ):

$$
c_{i_{1} \ldots i_{k}+1 \ldots i_{p} \ldots i_{N}}^{j}-c_{i_{1} \ldots i_{k} \ldots i_{p} \ldots i_{N}}^{j}=c_{i_{1} \ldots i_{k}+1 \ldots i_{p}+1 \ldots i_{N}}^{j}-c_{i_{1} \ldots i_{k} \ldots i_{p}+1 \ldots i_{N}}^{j}
$$

Then we rewrite them as:

$$
c_{i_{1} \ldots i_{k}+1 \ldots i_{p}+1 \ldots i_{N}}^{j}=c_{i_{1} \ldots i_{k} \ldots i_{p}+1 \ldots i_{N}}^{j}+c_{i_{1} \ldots i_{k}+1 \ldots i_{p} \ldots i_{N}}^{j}-c_{i_{1} \ldots i_{k} \ldots i_{p} \ldots i_{N}}^{j}
$$

These are recurrent formulae computing the values of all $c_{i_{1} \ldots i_{N}}^{j}$ from those computed initially by propagating from $c_{p_{1} \ldots p_{N}}^{j}$ in all diagonal directions. I leave the tedious details out. Once all $c_{i_{1} \ldots i_{N}}^{j}$ are computed, the matrix $\widehat{M}$ is determined. By construction it satisfies conditions $C_{1}^{N}$ and $C_{2}^{N}$ of Theorem 3 , hence it can be obtained from $M$ by a GOI-transformation. The uniqueness of $\widehat{M}$ follows immediately.

Example 3. Consider a three-person game with players $A_{1}, A_{2}, A_{3}$ of dimension $3 \times 3 \times 2$, with $2 D$ matrix-slices for the two actions of player $A_{3}$ as follows:

$$
M_{1}=\left[\begin{array}{c|ccc}
A_{1} \backslash A_{2} & A_{211} & A_{221} & A_{231} \\
\hline A_{111} & 1,2,0 & 2,3,1 & 3,1,2 \\
A_{121} & 2,3,3 & 3,4,4 & 4,2,5 \\
A_{131} & 6,5,6 & 7,6,7 & 5,7,8
\end{array}\right] \quad M_{2}=\left[\begin{array}{c|ccc}
A_{1} \backslash A_{2} & A_{212} & A_{222} & A_{232} \\
\hline A_{112} & 1,1,8 & 2,2,7 & 3,3,6 \\
A_{122} & 1,2,5 & 2,3,4 & 3,4,3 \\
A_{132} & 2,1,2 & 3,2,1 & 1,3,0
\end{array}\right]
$$


Suppose the tuple of desired entries in the transformed matrix is given with respect to the outcome with coordinates $(2,2,1)$ as follows:

$$
\widehat{M}_{1}^{0}=\left[\begin{array}{c|ccc}
A_{1} \backslash A_{2} & A_{211} & A_{221} & A_{231} \\
\hline A_{111} & & 1,2,3 & \\
A_{121} & 4,4,0 & 5,1,5 & 3,4,4 \\
A_{131} & & 8,4,8 &
\end{array}\right] \widehat{M}_{2}^{0}=\left[\begin{array}{cc|ccc}
A_{1} \backslash A_{2} & A_{212} & A_{222} & A_{232} \\
\hline A_{112} & & & \\
A_{122} & & 3,3,3 & \\
A_{132} & & &
\end{array}\right]
$$

Note that condition $C_{1}^{N}$ of Theorem 3 is satisfied.

The corresponding partial slices of the matrix of differences $c_{i_{1} i_{2} i_{3}}^{j}=\hat{a}_{i_{1} i_{2} i_{3}}^{j}-a_{i_{1} i_{2} i_{3}}^{j}$ are:

$$
C_{1}^{0}=\left[\begin{array}{c|ccc}
A_{1} \backslash A_{2} & A_{211} & A_{221} & A_{231} \\
\hline A_{111} & & -1,-1,2 & \\
A_{121} & 2,1,-3 & 2,-3,1 & -1,2,-1 \\
A_{131} & & 1,-2,1 &
\end{array}\right] C_{2}^{0}=\left[\begin{array}{cccc}
A_{1} \backslash A_{2} & A_{212} & A_{222} & A_{232} \\
\hline A_{112} & & & \\
A_{122} & & 1,0,-1 & \\
A_{132} & & &
\end{array}\right]
$$

The remaining entries of that matrix are then computed consecutively by using the identities

$$
\begin{aligned}
& c_{i_{1}+1 i_{2}+1 i_{3}}^{j}=c_{i_{1}+1 i_{2} i_{3}}^{j}+c_{i_{1} i_{2}+1 i_{3}}^{j}-c_{i_{1} i_{2} i_{3}}^{j} \\
& c_{i_{1} i_{2}+1 i_{3}+1}^{j}=c_{i_{1} i_{2} i_{3}+1}^{j}+c_{i_{1} i_{2}+1 i_{3}}^{j}-c_{i_{1} i_{2} i_{3},}^{j} \\
& c_{i_{1}+1 i_{2} i_{3}+1}^{j}=c_{i_{1} i_{2} i_{3}+1}^{j}+c_{i_{1}+1 i_{2} i_{3}}^{j}-c_{i_{1} i_{2} i_{3}}^{j},
\end{aligned}
$$

for each $j=1,2,3$. Thus, we first obtain

$$
C_{1}=\left[\begin{array}{c|ccc}
A_{1} \backslash A_{2} & A_{211} & A_{221} & A_{231} \\
\hline A_{111} & -1,3,-2 & -1,-1,2 & -4,4,0 \\
A_{121} & 2,1,-3 & 2,-3,1 & -1,2,-1 \\
A_{131} & 1,2,-3 & 1,-2,1 & -2,3,-1
\end{array}\right]
$$

then:

$$
C_{2}^{1}=\left[\begin{array}{c|ccc}
A_{1} \backslash A_{2} & A_{212} & A_{222} & A_{232} \\
\hline A_{112} & & -2,2,0 & \\
A_{122} & 1,4,-5 & 1,0,-1 & -2,5,-3 \\
A_{132} & & 0,1,-1 &
\end{array}\right]
$$

and finally:

$$
C_{2}=\left[\begin{array}{c|ccc}
A_{1} \backslash A_{2} & A_{212} & A_{222} & A_{232} \\
\hline A_{112} & -2,6,-4 & -2,2,0 & -5,7,-2 \\
A_{122} & 1,4,-5 & 1,0,-1 & -2,5,-3 \\
A_{132} & 0,5,-5 & 0,1,-1 & -3,6,-3
\end{array}\right]
$$

Eventually, we obtain the 2-dimensional matrix-slices of $\widehat{M}$ for the two actions of player $A_{3}$ :

$$
\begin{gathered}
\widehat{M}_{1}=\left[\begin{array}{c|ccc}
A_{1} \backslash A_{2} & A_{211} & A_{221} & A_{231} \\
\hline A_{111} & 0,5,-2 & 1,2,3 & -1,5,2 \\
A_{121} & 4,4,0 & 5,1,5 & 3,4,4 \\
A_{131} & 7,7,3 & 8,4,8 & 3,10,7
\end{array}\right] \\
\widehat{M}_{2}=\left[\begin{array}{c|ccc}
A_{1} \backslash A_{2} & A_{212} & A_{222} & A_{232} \\
\hline A_{112} & -1,7,4 & 0,4,7 & -2,10,4 \\
A_{122} & 2,6,0 & 3,3,3 & 1,9,0 \\
A_{132} & 2,6,-3 & 3,3,0 & -2,9,-3
\end{array}\right]
\end{gathered}
$$


In summary, just like in the case of two-person games, Theorems 3 and 4 together characterize precisely the payoff matrices $\widehat{M}$ that can be obtained from matrix $M$ by a GOItransformation; namely, any of them can be obtained by choosing a strategy profile in $M$ and setting suitable payoffs satisfying condition $C_{1}^{N}$ for all outcomes obtained by allowing any one, and only one, player to deviate from that strategy profile. Then, all payoffs in $\widehat{M}$ are computed by using the recurrent formulae derived from condition $C_{2}^{N}$.

\section{Conclusions}

The main contributions of the present paper are explicit and easy to apply, and they use characterizations of the game matrix transformations that can be induced by preplay offers for payments (or threats for punishments) in multiplayer normal form games. I have not considered here rationality issues that would determine which of these transformations can be affected by offers made by rational players. These are analyzed in the companion working paper in preparation [2]. Still, I argue that the results presented here are of direct game-theoretic relevance, because they can be used by the players to determine what mutually desirable transformed games (e.g., having dominant strategy equilibria with Pareto optimal outcomes) they can achieve by exchange of preplay offers, and then to search-by using the computational procedures that can be extracted from our proofsfor suitable offers that would induce the necessary game matrix transformations leading to the desired outcomes. Besides, the GOI-transformations of game payoff matrices and the groups of such transformations introduced here appear to be interesting algebraic constructs in their own right, worthy of further study.

Funding: This research received no external funding.

Data Availability Statement: Not applicable.

Acknowledgments: I thank the anonymous reviewers for the useful comments and corrections.

Conflicts of Interest: The author declares no conflict of interest.

\section{References}

1. Goranko, V.; Turrini, P. An offer you cannot refuse: Obtaining efficiency and fairness in preplay negotiation games with conditional offers. In Proceedings of the Fourth International Workshop on Logic, Rationality and Interaction (LORI'2013), Hangzhou, China, 9-12 October 2013; Huang, H., Grossi, D., Roy, O., Eds.; Springer: Berlin/Heidelberg, Germany, 2013; Volume 8196, pp. 110-123.

2. Goranko, V. Preplay Negotiations by Unconditional Offers in 2-Player Strategic Games: Power and Weakness. Working Paper, Presented at LOFT 2016. Available online: https://www2.philosophy.su.se/goranko/papers/LOFT2016PreplayNegotiationsUnconditionalOffers.pdf (accessed on 3 June 2016).

3. Goranko, V.; Turrini, P. Two-Player Preplay Negotiation Games with Conditional Offers. IGTR 2016, 18, 1550017. [CrossRef]

4. Nash, J.F., Jr. The Bargaining Problem. Econometrica 1950, 18, 155-162. [CrossRef]

5. Harsanyi, J.C. A Simplified Bargaining Model for the n-Person Cooperative Game. Int. Econ. Rev. 1963, 4, 194-220. [CrossRef]

6. Hart, S.; Mas-Colell, A. (Eds.) Cooperation: Game-Theoretic Approaches; Nato ASI Subseries F; Springer: Berlin/Heidelberg, Germany, 1997; Volume 155.

7. Rubinstein, A. Perfect Equilibrium in a Bargaining Model. Econometrica 1982, 50, 97-110. [CrossRef]

8. Osborne, M.; Rubinstein, A. Bargaining and Markets; Academic Press: San Diego, CA, USA, 1990.

9. Osborne, M.; Rubinstein, A. A Course in Game Theory; MIT Press: Cambridge, MA, USA, 1994.

10. Myerson, R. Game Theory: Analysis of Conflict; Harvard UP: Cambdridge, MA, USA, 1997.

11. Abreu, D.; Pearce, D. Bargaining, Reputation, and Equilibrium Selection in Repeated Games with Contracts. Econometrica 2007, 75, 653-710. [CrossRef]

12. Jackson, M.O; Wilkie, S. Endogenous Games and Mechanisms: Side Payments among Players. Rev. Econ. Stud. 2005, 72, 543-566. [CrossRef]

13. Yamada, A. Alternate contracts for side payments. Econ. Bull. 2005, 3, 1-5.

14. Ellingsen, T.; Paltseva, E. Confining the Coase theorem: Contracting, ownership, and free-riding. Rev. Econ. Stud. 2016, 83, 547-586. [CrossRef]

15. Strang, G. Introduction to Linear Algebra, 5th ed.; Wellesley-Cambridge Press: Cambridge, UK, 2016.

16. Fraleigh, J. A First Course in Abstract Algebra, 8th ed.; Pearson: London, UK, 2020. 